\title{
The Production of Hydroxyl Radicals in the Fetal Lamb Brain Resulting from Occlusion of the Umbilical Circulation and the Transplacental Effect of MCI-186 to Inhibit Hydroxyl Radical Production
}

\author{
YOSHIYUKI NAKAJIMA, NAOKI MASAOKA, YASUHITO HAYAKAWA, MASAO WATANABE, MASAJI NAGAISHI, \\ HISAEI SAKATA, AND TATSUO YAMAMOTO \\ Department of Obstetrics and Gynecology [Y.N., NM, Y.H., M.W., MN, Y.M.], Nihon University School of Medicine, Itabashi-Ku, Tokyo \\ 173-8610, Japan; Social Insurance Yokohama Central Hospital [H.S.], Naka-Ku, Yokohama 231-8553, Japan
}

\begin{abstract}
The present study evaluated hydroxyl radical production in fetal lamb brain during and after umbilical cord occlusion and examined the effects of injecting MCI-186 (3-metyl-1-phenyl-2pyrazolin-5-one; Edaravone), a hydroxyl radical scavenger, into the maternal circulation. In 11 chronically instrumented lambs, intermittent total umbilical cord occlusions $1 \mathrm{~min}$ out of every $3 \mathrm{~min}$ for $1 \mathrm{~h}$ and 10-min persistent total umbilical cord occlusion were performed with brain microdialysis using $5 \mathrm{mM}$ of sodium salicylate. In the remaining four lambs, $60 \mathrm{mg}$ of MCI-186 was administered into the maternal circulation from shortly before the end of 10 -min persistent total umbilical cord occlusion. Concentrations of 2,3-dihydroxybenzoic acid (2,3-DHBA), produced by hydroxyl radical reactions with sodium salicylate, were measured in perfusate by HPLC. Concentration of 2,3-DHBA in perfusate was $23.05 \pm 10.95 \mathrm{nM}$ before umbilical cord occlusion. Levels of 2,3-DHBA tended to increase slightly during and after intermittent umbilical cord occlusion, and were significantly increased by the end of 10-min occlusion (40.06 \pm $21.36 \mathrm{nM})$ and after occlusion (93.74 $\pm 29.17 \mathrm{nM})$. Infusion of MCI-186 suppressed 2,3-DHBA concentration to $29.35 \pm 14.95 \mathrm{nM}$ after occlusion. Administration of MCI-186 into the maternal circulation reduces hydroxyl radical production induced by umbilical cord occlusion in the fetal lamb brain. (Pediatr Res 59: 216-220, 2006)
\end{abstract}

$\mathrm{A}^{\mathrm{b}}$ bout $10 \%$ of cerebral palsy (CP) is due to asphyxia of the fetus/neonate at the time of delivery and it has been suggested that some cerebral neuropathy may have already been present in the fetus before the onset of labor (1-2).

Clapp et al. demonstrated that intermittent partial occlusion of the umbilical circulation for $1 \mathrm{~min}$ of every $3 \mathrm{~min}$ for $2 \mathrm{~h}$ was associated with an $89 \%$ frequency of histologically confirmed brain damage confined to the cerebral white matter in chronically instrumented fetal lambs, without systemic evidence of progressive acidosis (3). They concluded that umbilical cord compromise plays a causal role in a specific type of antenatal CNS injury in fetal lambs during late gestation. In

Received June 14, 2005; accepted August 25, 2005.

Correspondence: Yoshiyuki Nakajima, M.D., Department of Obstetrics and Gynecology, Nihon University School of Medicine, 30-1 Oyaguchi-Kamimachi, Itabashi-Ku, Tokyo, 173-8610, Japan; e-mail: nakaj613@abeam.ocn.ne.jp

The present study was partially supported by a Grant for Scientific Research (Fundamental Research C(2)) from the Japanese Ministry of Education.

DOI: $10.1203 / 01 . p d r .0000196373 .52086 . c 2$ addition, De Haan $e t$ al. reported that brief repeated umbilical cord occlusion caused sustained cytotoxic cerebral edema and focal infarcts in near-term fetal lambs (4). On the other hand Mallard et al. reported that single 10-min total umbilical cord occlusion causes measurable cerebral damage, predominantly to the hippocampus $(5,6)$.

These findings suggest that spontaneous cord complications might cause transient fetal hypoxic-ischemic episodes that, although not severe enough to result in death, are sufficient to irreversibly damage critical areas of the developing brain.

We assumed that this model represented conditions seen in repetitive hypoxic/ischemic-reperfusion reactions (7). Although reperfusion is necessary to prevent additional damage to neuronal tissue, the return of oxygenated blood to previously ischemic brain tissue has been recognized as an important mechanism causing substantial additional brain injury, including damage to both the microcirculation and parenchyma of the brain. This insult is dependent on free radical formation during posthypoxic/ischemic reoxygenation $(8,9)$. Damaging amounts of superoxide, hydrogen peroxide and hydroxyl radicals are generated by FFA and prostaglandin metabolism (10). Moreover, metabolites of hypoxanthine, accumulated in the brain during hypoxia and ischemia, and by xanthine oxidase, which is concentrated within the endothelium of the cerebral microvessels, lead to additional production of superoxide and hydroxyl radicals (11).

We have previously used the same experimental model as Clapp et al., implanting brain microdialysis probes into the cerebral white matter of fetal lambs to demonstrate that large quantities of oxygen free radicals result from increased conversion of hypoxanthine to xanthine generated in perfusate, without causing any marked degree of acidosis in the fetus during intermittent umbilical cord occlusion (7).

Numerous experimental approaches have obtained significant neuronal protection, and free radical scavengers capable of inhibiting oxidative injury are expected to emerge as a new method for the treatment of ischemic diseases such as cerebral ischemia $(7,9)$.

Abbreviations: 2,3-DHBA, 2,3-dihydroxy-benzoic acid 
MCI-186 (3-metyl-1-phenyl-2-pyrazolin-5-one; Edaravone) is a powerful antioxidative radical scavenger, and is the only drug currently available in clinical practice for the treatment of cerebral infarction (12-15). This potent scavenger of hydroxyl radicals inhibits not only hydroxyl radicals, but also iron-induced peroxidative injuries $(16,17)$. Furthermore, many reports have shown the protective effects of MCI-186 against hypoxic/ischemic reperfusion injury in the heart (18), liver (19), spinal cord (20) and neonatal brain (16). We are thus particularly interested in evaluating the effects of MCI-186 on fetal ischemic brain damage. However, no data have been reported concerning placental transfer of MCI-186 and effects on the fetus. The purpose of this study was to evaluate the possibility of intrauterine treatment using MCI-186 given maternally to prevent hydroxyl radical production in the fetal lamb brain caused by umbilical cord compression, and whether we could to apply MCI-186 to perinatal human fetus and neonates with severe asphyxia in the future.

\section{METHODS}

Experimental animal model. Before the initiation of the study, all experimental protocols were approved by the Animal Experimental Ethics Committee at Nihon University School of Medicine.

Instrumented fetal lambs were prepared in 11 pregnant ewes. Production of hydroxyl radical and changes to $\mathrm{Po}_{2}$ in fetal lambs brain resulting from intermittent or persistent total occlusion of the umbilical circulation were studied in seven animals. Another four animals were selected for determination of the transplacental effects of MCI-186 on inhibition of hydroxyl radical production.

Preparation and measurement in animal experimental model. Pregnant ewes (mean gestational age at surgery, $127 \pm 2 \mathrm{~d}$; normal term gestation, $145 \mathrm{~d}$ ) were anesthetized by intramuscular injection of xylazine $\mathrm{HCl}$ (Celactal; Bayer, Tokyo, Japan). Each ewe was immobilized on an operating surface. Following normal sterile procedures, polyvinyl catheters (inner diameter, 1 $\mathrm{mm}$ ) were placed in the fetal carotid artery, jugular vein and trachea. An FEP-BDP-1-1000 brain microdialysis probe (Eicom, Kyoto, Japan) was placed in the cerebral white matter at the following location: $1.5 \mathrm{~cm}$ posterior to the superior orbital edge; $5 \mathrm{~mm}$ lateral to the midline, and $1 \mathrm{~cm}$ ventral to the surface of the skull. $\mathrm{A} \mathrm{Po}_{2}-100 \mathrm{DW} \mathrm{O}$ probe $(21,22)$ (Inter Medical Co., Nagoya, Japan; polarographic oxygen electrodes; external diameter, $0.22 \mathrm{~mm}$ ) was placed in the contralateral hemisphere of the fetal brain. In addition, an inflatable occluder was placed, loosely encompassing the umbilical cord. Finally, a polyvinyl catheter was inserted into the maternal femoral vein and artery and advanced cephalad into the inferior vena cava. Catheters were exteriorized through a maternal flank incision. After surgery, animals were administered ampicillin trihydrate and gentamicin. Recovery from anesthesia was rapid, and ewes were usually standing and feeding $\leq 1 \mathrm{~h}$ after skin closure. Continuous recording of fetal heart rate, blood pressure and respiratory activity, in addition to maternal arterial blood pressure and heart rate, commenced immediately after surgery. Fetal heart rate was obtained simultaneously with fetal blood pressure using an instantaneous cardiotachometer. The fetal and maternal vascular catheters and fetal tracheal catheter were connected to appropriate pressure transducers. All signals were continuously displayed on a multi-channel polygraph recorder (RECTI-HORIZ-8 K; SanEi, Tokyo, Japan).

All 11 fetuses and their mothers exhibited complete recovery from surgical procedures and anesthesia within $24 \mathrm{~h}$, as judged by biochemical and biophysical parameters.

Study design, data collection and analysis. On post-operative day 1, after ensuring that both mothers and fetuses were physiologically normal and stable, perfusion of the microdialysis system was started and umbilical cord occlusion performed.

Using three established instrumented fetal lambs, intermittent total umbilical cord occlusion was performed $1 \mathrm{~min}$ out of every $3 \mathrm{~min}$ for $1 \mathrm{~h}$ with brain microdialysis using $5 \mathrm{mM}$ of sodium salicylate. Microdialysis perfusate was collected 30 min before starting occlusion, during 1-h intermittent occlusion and at $30 \mathrm{~min}$ after ending intermittent occlusion. In another 4 established instrumented fetal lambs, 10-min persistent total umbilical cord occlusion with brain microdialysis was performed. Perfusate was collected $30 \mathrm{~min}$ before starting occlusion, during 10-min occlusion and at 30 min after ending occlusion. $\mathrm{PO}_{2}$ levels in the deep white matter of fetal lambs brain were observed throughout the experimental period using a $\mathrm{PO}_{2}$ monitor $\left(\mathrm{PO}_{2}-100\right.$, Intermedical, Tokyo, Japan).

On the basis of the results of above mentioned experiments, in another four cases we administered $60 \mathrm{mg}$ of MCI-186 through the maternal femoral vein just before 10-min occlusions over a period of $10 \mathrm{~min}$ to check the inhibition of hydroxyl radical production. Dosage of MCI-186 was fixed at $60 \mathrm{mg}$, the current standard daily clinical dose for adults undergoing treatment for cerebral infarction. Considering the weight of a pregnant ewe, we selected a dose of $60 \mathrm{mg}$ of MCI-186 for administration into the maternal circulation. Microdialysis perfusate was collected at $30 \mathrm{~min}$ before starting occlusion, during 10-min occlusion and at $30 \mathrm{~min}$ after ending occlusion.

Concentrations of 2,3-dihydroxy-benzoic acid (2,3-DHBA), which results from hydroxyl radicals reacting with sodium salicylate, were measured in perfusate using HPLC (HPLC system LC10AD; column oven CTO10A, Shimadzu, Tokyo, Japan; Electro chemical detector nanospace SI-2, Shiseido, Tokyo, Japan; column, RIX RP-18T; f $2.0 \times 250 \mathrm{~mm}$; column temperature, $30^{\circ} \mathrm{C}$; flow rate, $0.2 \mathrm{~mL} / \mathrm{min}$; mobile phase, $0.1 \mathrm{M}$ phosphate buffer, $\mathrm{pH} 2.8$ ).

All values are expressed as mean $\pm \mathrm{SD}$. Comparisons between groups were made using unpaired $t$-tests. Values of $p<0.05$ were considered statistically significant.

\section{RESULTS}

$\mathrm{Po}_{2}$ levels during intermittent obstruction. Fig. 1 shows $\mathrm{PO}_{2}$ levels in the deep white matter of a fetal lamb brain during intermittent obstruction of the umbilical circulation. $\mathrm{Po}_{2}$ decreased slightly from $19.8 \pm 3.2$ to $15.9 \pm 2.1 \mathrm{~mm} \mathrm{Hg}$ in the deep white matter of the fetal brain during and shortly after intermittent umbilical cord occlusion.

$\mathrm{Po}_{2}$ levels during persistent obstruction. Fig. 2 shows $\mathrm{Po}_{2}$ levels in the deep white matter of a fetal lamb brain during 10-min persistent obstruction of the umbilical circulation. $\mathrm{Po}_{2}$ decreased significantly from $21.4 \pm 2.6 \mathrm{~mm} \mathrm{Hg}$ before occlusion to $4.4 \pm 1.6 \mathrm{~mm} \mathrm{Hg}$ during 10 -min occlusion. This low $\mathrm{PO}_{2}$ persisted after cessation of occlusion and gradually returned to pre-occlusion levels.

Concentrations of 2,3-DHBA before, during and after intermittent occlusion. Fig. 3 shows 2,3-DHBA concentrations in fetal brain microdialysis perfusates before, during and after intermittent occlusion of the umbilical circulation. Concentration of 2,3-DHBA in perfusate was $23.05 \pm 10.95 \mathrm{nM}$ before intermittent umbilical cord occlusion, displaying a slight tendency to increase during occlusion.

Concentrations of 2,3-DHBA before, during and after persistent occlusion. Fig. 4 shows 2,3-DHBA concentrations in fetal brain microdialysis perfusates before, during and after persistent occlusion of the umbilical circulation. A significant

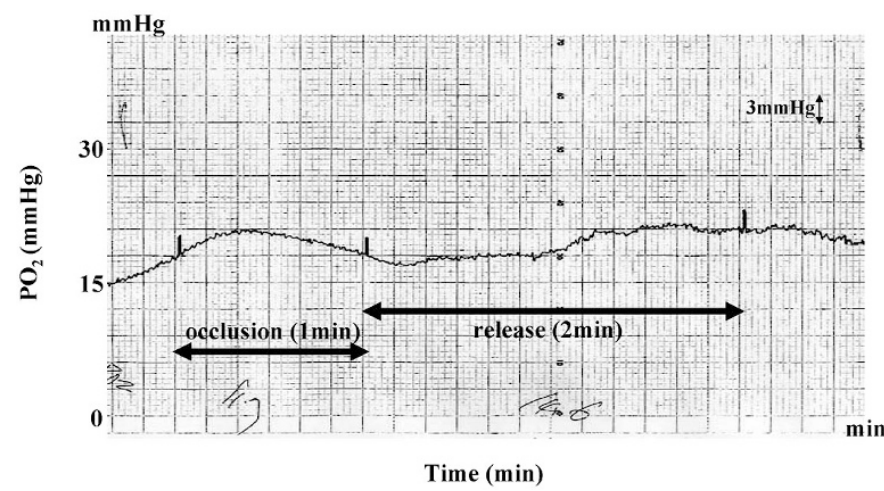

Figure 1. Changes in $\mathrm{Po}_{2}$ in the deep white matter of fetal lamb brains during intermittent obstruction of the umbilical circulation. 


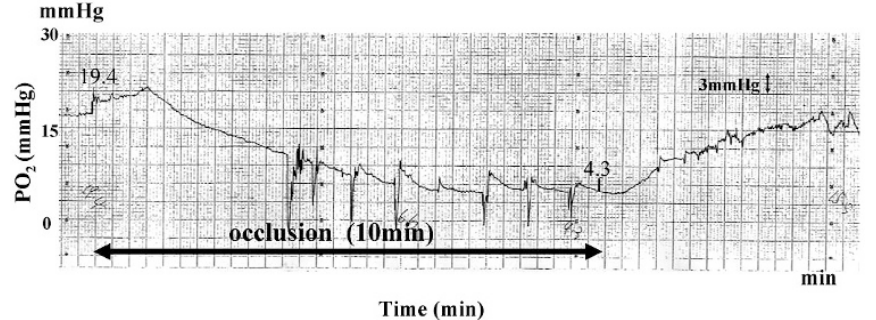

Figure 2. Changes in $\mathrm{Po}_{2}$ in the deep white matter of fetal lamb brains during persistent obstruction of the umbilical circulation.

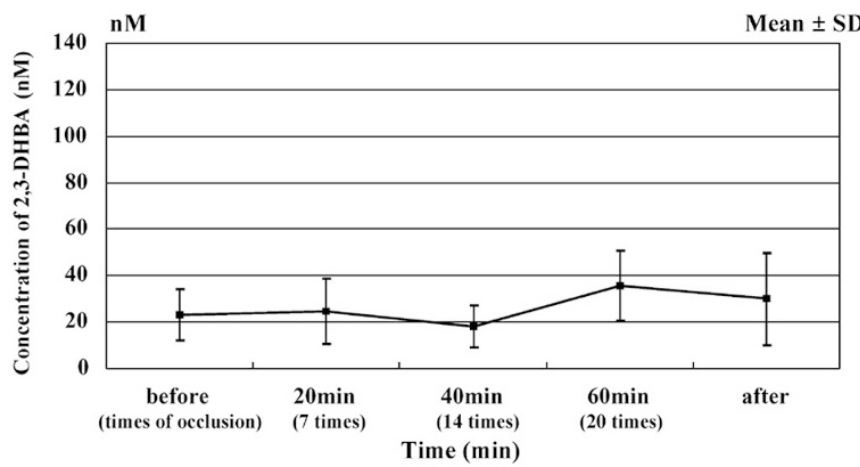

Figure 3. Concentrations of 2,3-DHBA in fetal brain microdialysis perfusates before, during and after intermittent occlusion of the umbilical circulation. Concentration of 2,3-DHBA tended to increase slightly during intermittent umbilical cord occlusion (not significant).

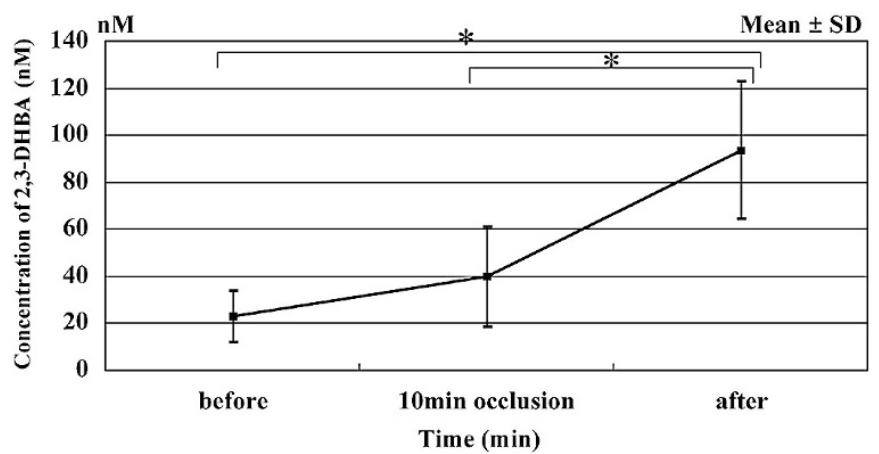

Figure 4. Concentration of 2,3-DHBA in fetal brain microdialysis perfusates before, during and after persistent occlusion of umbilical circulation. Concentration of 2,3-DHBA was significantly increased at the end and $30 \mathrm{~min}$ after 10 -min occlusion $(* p<0.05)$

increase in 2,3-DHBA concentration was noted both at the end of 10 -min occlusion $(40.06 \pm 21.36 \mathrm{nM} ; p<0.05)$ and at 30 min after the end of occlusion $(93.74 \pm 29.17 \mathrm{nM} ; p<0.05)$.

Concentrations of 2,3-DHBA before, during and after persistent occlusion with addition of MCI-186. Fig. 5 shows 2,3-DHBA concentrations in fetal brain microdialysis perfusates before, during and after 10-min persistent occlusion of the umbilical circulation with the addition of MCI-186 to the maternal circulation. Infusion of MCI-186 into the maternal circulation resulted in significant suppression of 2,3-DHBA concentration to $29.35 \pm 14.95 \mathrm{nM}$ at $30 \mathrm{~min}$ after the end of occlusion $(p<0.05)$. This value was significantly lower than that seen after occlusion without MCI-186 (93.74 \pm 29.17 $\mathrm{nM} ; p<0.05)$.

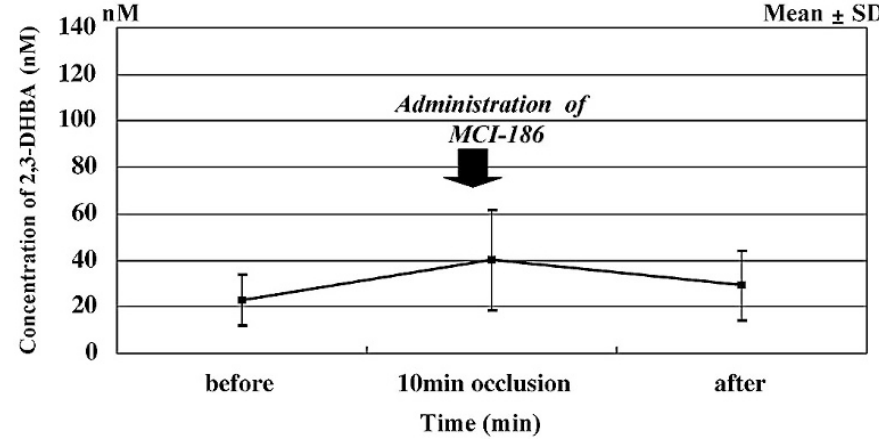

Figure 5. Concentration of 2,3-DHBA in fetal brain microdialysis perfusates before, during and after persistent occlusion of the umbilical circulation after introducing MCI-186 into the maternal circulation. Concentrations of 2,3DHBA were not increased at the end or $30 \mathrm{~min}$ after the end of 10-min occlusion $(* p<0.05)$.

\section{DISCUSSION}

Production of oxygen free radicals may represent the background of ischemic/hypoxic reperfusion injury in the fetal CNS $(7-9,23,24)$. MCI-186, which acts as a potent scavenger of hydroxyl radicals and inhibits not only hydroxyl radicals but also iron-induced peroxidative injuries, may thus help to prevent the onset of brain damage due to ischemic/hypoxic reperfusion (12-17).

Numerous reports have discussed the use of MCI-186 to treat the brain and other organs after ischemic/hypoxic insult $(16,18-20)$. From the perspective of the mechanisms of action, MCI-186 should be administered during ischemia/ hypoxia or as early as soon as possible in reperfusion. Delayed administration of MCI-186 is considered ineffective. Theoretically, administration of MCI-186 to the asphyxiated fetus in utero is ideal. With that in mind, placental and fetal bloodbrain barrier transfer of MCI-186 administered maternally is very important, but few reports have discussed this method.

In our study, brain microdialysis was adopted to evaluate hydroxyl radical production caused by umbilical cord occlusion and MCI-186 transfer from the maternal circulation to fetal cerebral neurocytes. To ensure the recovery of maternal animals and fetuses from anesthesia and surgical procedures and to establish conditions for measurement by brain microdialysis at a constant level, the experiment was performed on post-operative day one. Although microdialysis probes used in the experiment had an external diameter of only $0.22 \mathrm{~mm}$ at the dialysis membrane, injury to cerebral tissues was inevitable, and commencement of the study had to wait until recovery. In addition, efficiency of dialysis decreases when gliosis becomes prominent during the restoration process, and bias may have occurred in the measurement of substances at low concentrations.

Recently, in vivo formation of free radicals in cerebral extracellular fluid has been identified by spin trapping and electron spin resonance techniques coupled with cerebral microdialysis. This study adopted the conversion of salicylate to 2,3-DHBA as an estimate of hydroxyl radical production $(25,26)$. Hydroxyl radicals cause salicylate to form $2,3-$ and 2,5-DHBA, and a small amount of catechol (27-29). Levels of 2,3-DHBA represent a more reliable marker of hydroxyl 
radical reactions, as 2,5-DHBA can also be generated by enzymatic reactions, such as from the cytochrome P450 system.

Using oxygen electrodes, low $\mathrm{Po}_{2}$ and reoxygenation were confirmed in fetal brain tissues during and after umbilical cord occlusion. Changes in $\mathrm{Po}_{2}$ during 10-min persistent occlusion were the same as reported by Sakata et al. in a study of brainstem $\mathrm{PO}_{2}$ following umbilical cord occlusion in full-term rat fetuses using the same oxygen electrodes (30).

Regarding 2,3-DHBA concentrations in fetal brain microdialysis perfusate, a significant increase was recognized with 10-min persistent occlusion, but no obvious increase was noted with intermittent occlusion. With intermittent umbilical cord occlusion, large amounts of oxygen free radicals have been observed to be released into fetal brain tissue by measuring the chemiluminescence generated by mixing collected fetal brain microdialysis perfusates containing superoxide with a cypridina luciferin analogue (7). Intensity of chemiluminescence during intermittent umbilical cord occlusion was about double that for nonspecific chemiluminescence. The reason for the lack of any apparent increase in 2,3-DHBA levels in the present study, and thus presumably a lack of hydroxyl radical production, was unclear. Differing degrees of hypoxia may have been created in the fetal brain between persistent and intermittent occlusion. Alternatively, we have previously described a gradual decrease in NO levels from NO electrodes placed in fetal brain white matter during intermittent partial umbilical cord occlusion (31). This phenomenon might mean that $\mathrm{NO}$ and superoxide react rapidly and generate peroxinitrite. As a result, hydroxyl radicals might not increase during and after intermittent umbilical cord occlusion.

MCI-186 is unstable in biologic matrix such as blood or plasma due to its radical scavenger function, but is stable in organic solvent. Because MCI-186 should be rapidly extracted with organic solvent after immediate preparation of plasma to determine MCI-186 concentrations in plasma, we could not measure the MCI- 186 concentration directly in the fetal blood and fetal brain microdialysis perfusate.

Dosage of MCI-186 was fixed at $60 \mathrm{mg}$, the current standard daily clinical dose for adults undergoing treatment for cerebral infarction. Considering the weight of a pregnant ewe, we selected a dose of $60 \mathrm{mg}$ of MCI-186 for administration into the maternal circulation over a period of $10 \mathrm{~min}$, starting just before the end of umbilical cord occlusion.

A massive increase in hydroxyl radical concentration after 10-min persistent umbilical cord occlusion was confirmed. MCI-186 administered to the pregnant ewe was then able to pass the placenta and blood-brain barrier, and inhibit hydroxyl radical production in the fetal lamb brain due to hypoxiareperfusion induced by umbilical cord occlusion.

In this experiment, compression of the umbilical cord was considered equivalent to compression caused by uterine contractions during premature labor. Recent attention has been focused on periventricular leukomalacia (PVL) in low birthweight neonates as a cause of cerebral palsy. In cases of PVL, variable transient bradycardia (due to umbilical cord compression, and thus representing an umbilical factor) reportedly appears at high frequency in antepartum fetuses or during delivery (32). However, variable transient bradycardia during delivery is often observed in routine clinical practice, and is thus very unlikely to immediately induce organ disorder. Any such damage would presumably depend on factors such as the degree and duration of cord occlusion. Furthermore, cerebral neuropathy frequently develops in cases of premature birth, and this is attributed to structural prematurity of the fetal cerebral blood vessels, that is, the presence of a low-perfusion area in the periventricular region due to immature development of the ventriculopetal and ventriculofugal blood vessels, or to immaturity of autoregulation in the cerebral blood vessels (33).

Although morphologic differences exist between the placenta in humans and sheep, the present results demonstrated good transfer of MCI-186 from mother to fetus and adequate inhibition of hydroxyl radical production in the fetal brain. However, pathologic evaluation of fetal brains and a check of dosage, administration time and adverse effects to mother and fetus for MCI-186 must be performed, these results suggest the possibility of intrauterine management to reduce fetal brain damage caused by hydroxyl radicals synthesized by umbilical cord compression during delivery.

\section{REFERENCES}

1. Freeman JM, Nelson KB 1988 Intrapartum asphyxia and cerebral palsy. Pediatrics 82:240-249

2. Blair E, Stanley FJ 1988 Intrapartum asphyxia: a rare cause of cerebral palsy J Pediatr 112:515-519

3. Clapp JF, Peress NS, Wesley M, Mann LI 1988 Brain damage after intermittent partial cord occlusion in the chronically instrumented fetal lamb. Am J Obstet Gynecol 159:504-509

4. De Haan HH, Gunn AJ, Williams CE, Gluckman PD 1997 Brief repeated umbilical cord occlusions cause sustained cytotoxic cerebral edema and focal infarcts in near-term fetal lambs. Pedatr Res 41:96-104

5. Mallard EC, Williams CE, Johnston BM, Gluckman PD 1994 Increased vulnerability to neuronal damage after umbilical cord occlusion in fetal sheep with advancing gestation. Am J Obstet Gynecol 170:206-214

6. Mallard EC, Gunn AJ, Williams CE, Johnston BM, Gluckman PD 1992 Transient umbilical cord occlusion causes hippocampal damage in the fetal sheep. Am J Obstet Gynecol 167:1423-1430

7. Masaoka N, Hayakawa Y, Ohgame S, Sakata H, Satoh K, Takahashi H 1998 Changes in purine metabolism and production of oxygen free radicals by intermittent partial umbilical cord occlusion in chronically instrumented fetal lambs. J Obstet Gynecol Res 24:63-71

8. Palmer C 1995 Hypoxic-ischemic encephalopathy. Therapeutic approaches against microvascular injury, and role of neutrophils, PAF, and free radicals. Clin Perinatol 22:481-517

9. Buonocore G, Perrone S, Bracci R 2001 Free radicals and brain damage in the newborn. Biol Neonate 79:180-186

10. Pourcyrous M, Leffler CW, Bada HS, Korones SB, Busija DW 1993 Brain superoxide anion generation in asphyxiated piglets and the effect of indomethacin at therapeutic dose. Pediatr Res 34:366-369

11. Betz AL 1985 Identification of hypoxanthine transport and xanthine oxidase activity in brain capillaries. J Neurochem 44:574-579

12. Edaravone Acute Infarction Study Group 2003 Effect of a novel free radical scavenger, edaravone (MCI-186), on acute brain infarction. Randomized, placebocontrolled, double-blind study at multicenters. Cerebrovasc Dis 15:222-229.

13. Wu TW, Zeng LH, Wu J, Fung KP 2000 MCI-186: further histochemical and biochemical evidence of neuroprotection. Life Sci 67:2387-2392

14. Mizuno A, Umemura K, Nakashima M 1998 Inhibitory effect of MCI-186, a free radical scavenger, on cerebral ischemia following rat middle cerebral artery occlusion. Gen Pharmacol 30:575-578

15. Yamamoto T, Yuki S, Watanabe T, Mitsuka M, Saito KI, Kogure K 1997 Delayed neuronal death prevented by inhibition of increased hydroxyl radical formation in a transient cerebral ischemia. Brain Res 11:240-242

16. Yasuoka N, Nakajima W, Ishida A, Takada G 2004 Neuroprotection of edaravone on hypoxic-ischemic brain injury in neonatal rats. Brain Res Dev Brain Res 19:129-139

17. Watanabe K, Morinaka Y, Iseki K, Watanabe T, Yuki S, Nishi H 2003 Structureactivity relationship of 3-methyl-1-phenyl-2-pyrazolin-5-one (edaravone). Redox Rep 8:151-155

18. Tsujita K, Shimomura H, Kawano H, Hokamaki J, Fukuda M, Yamashita T, Hida S, 
Nakamura Y, Nagayoshi Y, Sakamoto T, Yoshimura M, Arai H, Ogawa H 2004 Effects of edaravone on reperfusion injury in patients with acute myocardial infarction. Am J Cardiol 15:481-484

19. Abe T, Unno M, Takeuchi H, Kakita T, Katayose Y, Rikiyama T, Morikawa T, Suzuki M, Matsuno S 2004 A new free radical scavenger, edaravone, ameliorates oxidative liver damage due to ischemia-reperfusion in vitro and in vivo. J Gastrointest Surg 8:604-615

20. Takahashi G, Sakurai M, Abe K, Itoyama Y, Tabayashi K 2004 MCI-186 reduces oxidative cellular damage and increases DNA repair function in the rabbit spinal cord after transient ischemia. Ann Thorac Surg 78:602-607

21. Nakamura S, Arakawa S, Nishiike S 1995 A long-lasting change in the excitability of fetal brain neurons following activation of dam's hypothalamus in rats. Am J Physiol 269:R236-R244

22. Sakata Y, Fujioka T, Chowdhury GM, Nakamura S 2000 In vivo electrical activity of brainstem neurons in fetal rats during asphyxia. Brain Res 21:271-280

23. Castillo-Melendez M, Chow JA, Walker DW 2004 Lipid peroxidation, caspase-3 immunoreactivity, and pyknosis in late-gestation fetal sheep brain after umbilical cord occlusion. Pediatr Res 55:864-871

24. Peeters C, van Bel, F 2001 Pharmacotherapeutical reduction of post-hypoxicischemic brain injury in the newborn. Biol Neonate 79:274-280

25. Zini I, Tomasi A, Grimaldi R, Vannini V, Agnati LF 1992 Detection of free radicals during brain ischemia and reperfusion by spin trapping and microdialysis. Neurosci Lett 138:279-282
26. Matsuo Y, Kihara T, Ikeda M, Ninomiya M, Onodera H, Kogure K 1996 Role of platelet-activating factor and thromboxane $\mathrm{A} 2$ in radical production during ischemia and reperfusion of the rat brain. Brain Res 709:296-302

27. Zhang J, Piantadosi CA 1994 Prolonged production of hydroxyl radical in rat hippocampus after brain ischemia-reperfusion is decreased by 21 -aminosteroids. Neurosci Lett 177:127-130

28. Halliwell B 1992 Reactive oxygen species and the central nervous system. J Neurochem 59:1609-1623

29. Halliwell B, Kaur H, Ingelman-Sundberg M 1991 Hydroxylation of salicylate as an assay for hydroxyl radicals: a cautionary note. Free Radic Biol Med 10:439441

30. Sakata Y, Fujioka T,Inouye S, Endoh H, Nakamura S 2002 Paradoxical rise in brainstem $\mathrm{PO}(2)$ following umbilical cord occlusion in full-term rat fetuses. Neurosc Lett 330:167-170.

31. Hamano S, Masaoka N, Hayakawa Y, Ohgame S, Nakajima Y, Nagaishi M, Yamamoto T 2003 Changes of NO and oxygen levels in the brain of fetal lambs during intermittent and persistent obstruction of the umbilical circulation. J Perinatal Med 31:309_

32. Saliba E, Marret S 2001 Cerebral white matter damage in the preterm infant: pathophysiology and risk factors. Semin Neonatol 6:121-133

33. Takashima S, Tanaka K 1978 Development of cerebrovascular architecture and its relationship to periventricular leukomalacia. Arch Neurol 35:11-16 